Research Article

\title{
The Effects of Load Location on Dropper Stress in a Catenary System for a High-Speed Railway
}

\author{
Fan He ${ }^{10}{ }^{1,2}$ and Dandan Guo ${ }^{1}$ \\ ${ }^{1}$ School of Science, Beijing University of Civil Engineering and Architecture, Beijing 100044, China \\ ${ }^{2}$ Beijing Key Laboratory of Functional Materials for Building Structure and Environment Remediation, \\ Beijing University of Civil Engineering and Architecture, Beijing 100044, China \\ Correspondence should be addressed to Fan He; hefan@bucea.edu.cn
}

Received 6 July 2020; Revised 22 September 2020; Accepted 24 September 2020; Published 8 October 2020

Academic Editor: Ivan Giorgio

Copyright ( 2020 Fan He and Dandan Guo. This is an open access article distributed under the Creative Commons Attribution License, which permits unrestricted use, distribution, and reproduction in any medium, provided the original work is properly cited.

\begin{abstract}
In this paper, the effects of load location on dropper stress are studied. We treat contact wire as a beam element and derive its response equation and then deduce the stress equation of dropper. A computer code based on MATLAB is written to calculate dropper stress using the finite difference method. The results show that there are three stages during the period of the stress changes of dropper, including instant rebound, damped sinusoidal vibration, and bending compression. The shorter the distance away from a load is, the larger the vertical displacement of the dropper is, which results in the corresponding increases of its stress amplitude and the maximum tensile stress. The load location has a significant impact on the stress changes of dropper. Compared to the condition of the load in the middle, the load acting on the edge of contact wire could induce the larger tensile stress when both ends of contact wire are considered as free boundaries. Therefore, it is necessary to add supports at both ends.
\end{abstract}

\section{Introduction}

Dropper is an important part to improve the current collection and force performance of a catenary system, and it is one of the key parts to ensure the safety of electrified railway. With the increase of train speed, the fracture of dropper has often happened in recent years. Under the action of wind, the broken dropper is very easy to be entangled with contact wire, which will damage pantograph and contact wire and result in the failure of train operation. The working state of dropper depends on its stress. The investigation of dropper failure is of great significance to avoid the fracture occurrence and ensure the safe operation of high-speed railway.

There are many researches on a catenary structure. Cho established a finite element model of the interaction between pantograph and catenary and compared the simulation results with the actual measurement. The results show that the compression relaxation of dropper affects the dynamics between pantograph and catenary [1]. When a pantograph acts on a catenary, an impact load could be generated, which makes droppers subject to uneven axial impact force, resulting in different fatigue damage of droppers at different locations $[2,3]$. The fatigue effects of different pretension, bending load, axial load, and other factors on wire rope have been studied by finite element models [4-6]. By adjusting the length of dropper, the working quality of catenary could be improved without increasing supports, so as to minimize the fatigue fracture of catenary and dropper caused by an impact of pantograph [7]. It is found that the fatigue life of dropper decreases with the increase of vibration amplitude, frequency, and force [8]. In addition, many researchers used different simulation methods to analyze the dynamic performance of catenary and pantograph [9-14].

The reliability of dropper is directly related to the stress conditions it receives; however, there are few researches on dropper stress. Furthermore, when a load acts on different locations of contact wire, how dropper stress changes is also worthy of attention. It is very important for the driving safety. In fact, a catenary system can be associated to cable (or cable-strut) structures in civil and bridge engineering. 
There have been some related studies on stress design and structural analysis on novel cable (or cable-strut) structures in recent years [15-18]. Due to many similarities, the investigated results of a catenary system can be extended to other engineering fields. Thus, there are potential applications of the proposed calculation method of dropper stress. In this paper, we investigate the effects of a varying load location on dropper stress in a catenary structure, while providing the theoretical basis for the fatigue mechanism of dropper under a dynamic load. This work might also give a reference for the study of cable (or cable-strut) structures.

\section{Dynamic Equation of Contact Wire}

2.1. Catenary Model. In order to calculate the stresses of a span of five droppers when a load acts on different locations, a simple chain suspension catenary is considered in this paper. Its structure is symmetrical, and the distance between two adjacent droppers is $10 \mathrm{~m}$. The lengths of droppers I and $\mathrm{V}$ are $1.6 \mathrm{~m}$, and those of droppers II and IV are $1.295 \mathrm{~m}$, and that of dropper III is $1.2547 \mathrm{~m}$. The structure diagram is shown in Figure 1. The involved analysis can be further simplified by using symmetry analysis $[19,20]$.

2.2. Differential Equation of Contact Wire Motion. In this paper, the catenary model is established by a beam element, and the differential equation of contact wire motion is as follows:

$$
\mathrm{EI} \frac{y^{4}(x, t)}{\partial x^{4}}+\rho A \frac{\partial^{2} y(x, t)}{\partial t^{2}}=F(t) \delta(x-a),
$$

where $\rho$ is the density of contact wire, $A$ is the cross-sectional area, $\mathrm{EI}$ is the bending rigidity, $E$ is the elastic modulus, $I$ is the section moment of inertia, $y(x, t)$ is the vertical displacement, $F(t)$ is a dynamic load, and $a$ represents the distance between the load and the origin.

In order to solve the differential equation of contact wire, the solution of equation (1) can be set as follows by using the method of separating variables:

$$
y(x, t)=\sum_{i=1}^{\infty} Y_{i}(x) q_{i}(t),
$$

where $Y_{i}(x)$ is the modal function of the transverse vibration of a beam, which can be obtained by the free vibration of a beam and $q_{i}(t)$ is a sine function. The specific form is solved by the following procedure.

The free vibration equation of a beam is as follows:

$$
\mathrm{EI} \frac{\partial y^{4}(x, t)}{\partial x^{4}}+\rho A \frac{\partial^{2} y(x, t)}{\partial t^{2}}=0 .
$$

Let the solution of equation (3) be the following:

$$
y(x, t)=Y(x) \sin (\omega t+\phi) .
$$

Substituting equation (4) into equation (3), it can be converted into solving the following equation:

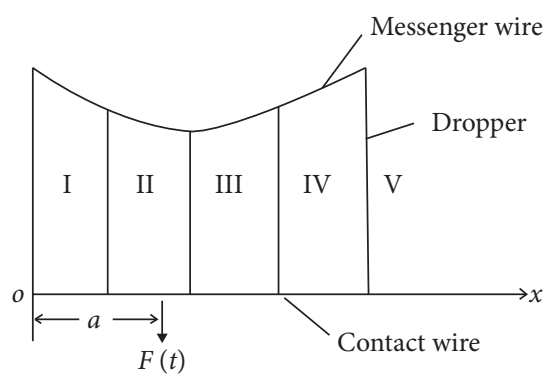

FIgURE 1: Force diagram of a span of five droppers under a load.

$$
Y^{4}(X)-k^{4} Y(x)=0,
$$

where $k^{4}=(\rho A / E I) \omega^{2}$.

The characteristic equation of equation (5) is as follows:

$$
\lambda^{4}-k^{4}=0 .
$$

Thus, its characteristic root is written as follows:

$$
\begin{aligned}
& \lambda_{1}=k, \\
& \lambda_{2}=-k, \\
& \lambda_{3}=k i, \\
& \lambda_{4}=-k i .
\end{aligned}
$$

However,

$$
\begin{aligned}
e^{ \pm k x} & =\cosh k x \mp \sinh k x, \\
e^{ \pm k i x} & =\cos k x \pm \sin k x .
\end{aligned}
$$

Then, the general solution of equation (5) can be expressed as a linear combination of $\cosh k x, \sinh k x, \cos k x$, and $\sin k x$. Therefore, it is as follows:

$$
Y(x)=A_{1} \sin k x+A_{2} \cos k x+A_{3} \sinh k x+A_{4} \cosh k x,
$$

where $A_{1}, A_{2}, A_{3}, A_{4}$ can be calculated by the boundary conditions at both ends of a beam.

In this mode, the contact wire is regarded as a beam with free ends; therefore, the bending moment and shear force at both ends of the contact wire are zero:

$$
\begin{aligned}
Y^{\prime \prime}(0) & =0, \\
Y^{\prime \prime}(l) & =0, \\
Y^{\prime \prime \prime}(0) & =0, \\
Y^{\prime \prime \prime}(l) & =0 .
\end{aligned}
$$

Hence, we can get the following expressions:

$$
\left\{\begin{array}{l}
A_{1}=A_{3}, \\
A_{2}=A_{4}, \\
A_{1}(\sinh k l-\sin k l)+A_{2}(\cosh k l-\cos k l)=0, \\
A_{1}(\cosh k l-\cos k l)+A_{2}(\sinh k l-\sin k l)=0,
\end{array}\right.
$$


where $A_{1}, A_{2}$ have non-zero solutions; thus, $\left|\begin{array}{cc}\sinh k l-\sin k l & \cosh k l-\cos k l \\ \cosh k l-\cos k l & \sinh k l+\sin k l\end{array}\right|=0$.

The frequency equation can be obtained as follows:

$$
\cos k l \cosh k l-1=0 .
$$

The solution is as follows:

$$
k_{i} l \approx\left(i+\frac{1}{2}\right) \pi
$$

Due to $k^{4}=(\rho A / E I) \omega^{2}$, then the natural frequency is $\omega_{i}=k_{i}^{2} \sqrt{(\mathrm{EI} / \rho A)}=\left((i+(1 / 2))^{2} \pi^{2} / l^{2}\right) \sqrt{(\mathrm{EI} / \rho A)}$.

The corresponding modal function is the following expression:

$$
Y_{i}(x)=\cosh k_{i} x+\cos k_{i} x-\frac{\cos k_{i} l-\cosh k_{i} l}{\sin k_{i} l-\sinh k_{i} l}\left(\sinh k_{i} x+\sin k_{i} x\right)
$$

When there is a load, the forced vibration equation of a beam is equation (1). It is required to solve $Y_{i}(x)$ and $q_{i}(t)$.

Substituting equation (2) and (14) into equation (1), the following equation can be written:

$$
\sum_{i=1}^{\infty}\left[\operatorname{EI}_{i}(t) Y_{i}^{4}(x)+\rho A Y_{i}(x) q^{2}(t)\right]=F(t) \delta(x-a) .
$$

Multiplying $Y_{j}(x)$ on both sides of equation (15) and integrating $x$ along the beam length, it can be obtained as follows:

$$
\sum_{i=1}^{n}\left[q_{i}(t) \int_{0}^{l} \operatorname{EIY}_{j}(x) Y_{i}^{4}(x) \mathrm{d} x+q_{i}^{2}(t) \int_{0}^{l} \rho A Y_{i}(x) Y_{j}(X) \mathrm{d} x\right]=\int_{0}^{l} F(t) \delta(x-a) Y_{j}(x) \mathrm{d} x
$$

Considering the regularity of modal function, the following expressions can be obtained:

$$
\begin{aligned}
& \int_{0}^{l} \rho A Y_{i}(x) Y_{j}(x) \mathrm{d} x=\delta_{i j}, \\
& \int_{0}^{l} \operatorname{EIY}_{j}(x) Y_{i}^{4}(x) \mathrm{d} x=\omega_{i}^{2} \delta_{i j} .
\end{aligned}
$$

Hence, we get the following:

$$
\omega_{i}^{2} q_{i}(t)+q_{i}^{2}(t)=F(t) Y_{i}(a) .
$$

Let $F(t)=F \sin \omega t$; then, the steady solution of the mode is as follows:

$$
q_{i}(t)=\frac{F Y_{i}(a)}{\omega_{i}^{2}\left[1-\left(\omega / \omega_{i}\right)^{2}\right]} \sin \omega t, \quad(i=1,2,3, \cdots)
$$

Thus, the response equation of the beam is expressed as follows:

$$
y(x, t)=\sum_{i=1}^{\infty} Y_{i}(x) q_{i}(t)=F \sin \omega t \sum_{i=1}^{\infty} \frac{Y_{i}(a) Y_{i}(x)}{\omega_{i}^{2}\left[1-\left(\omega / \omega_{i}\right)\right]}, \quad(i=1,2,3, \cdots)
$$

where $\omega_{i}$ is the natural frequency of the beam and the constant force $F$ and the frequency $f$ are, respectively, set to be $100 \mathrm{~N}$ and $1 \mathrm{~Hz}$; thus, $\omega=2 \pi f=2 \pi$.

It is well known that $i$ is larger, and the vibration of dropper is smaller. Therefore, the influence of higher order is very small, and then $i=5$ is selected here.

\section{Calculation of Dropper Stress}

3.1. Theoretical Equation. The section method is adopted, and the micro-element segment of a dropper is considered for stress analysis. The force analysis diagram is shown in Figure 2. 


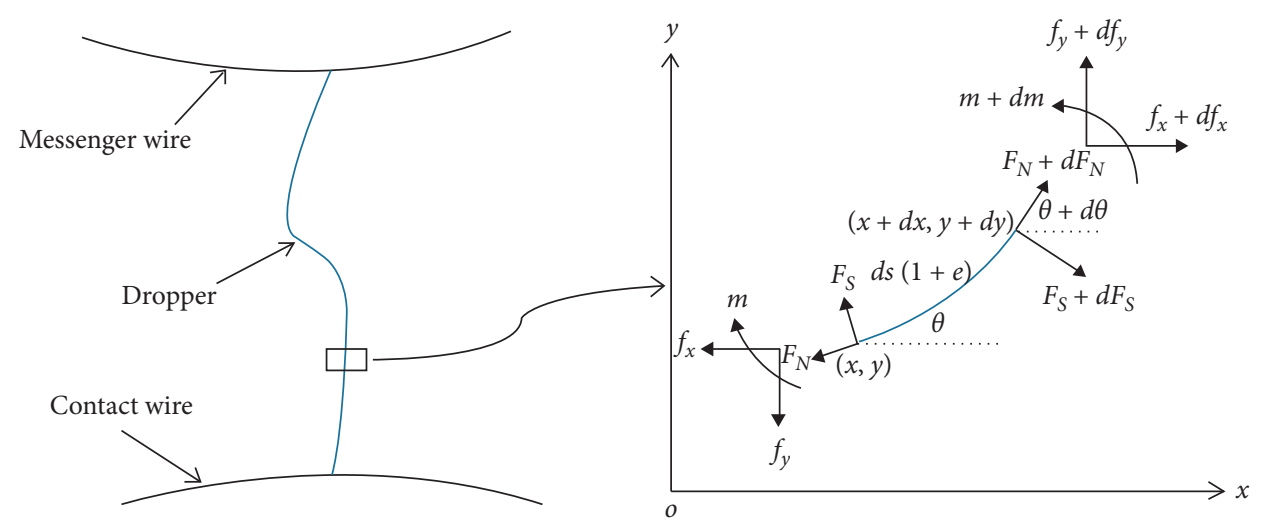

FIGURE 2: Force analysis diagram of the micro-element segment of a dropper.

In order to simplify the subsequent calculation, we refer to the following dimensionless variables:

$$
\begin{aligned}
\bar{x} & =\frac{x}{L_{d}}, \\
\bar{y} & =\frac{y}{L_{d}}, \\
\bar{v}_{x} & =\frac{v_{x}}{\sqrt{g L_{d}}}, \\
\bar{v}_{y} & =\frac{v_{y}}{\sqrt{g L_{d}}}, \\
\bar{f}_{x} & =\frac{f_{x}}{E_{d} A_{d}}, \\
\bar{m} & =\frac{m}{E_{d} A_{d} L_{d}}, \\
u & =\frac{\rho_{d} g L_{d}}{E_{d}}, \\
\bar{t} & =\frac{t}{\sqrt{\left(L_{d} / g\right)}}, \\
\lambda^{2} & =\frac{E_{d} A_{d} L_{d}^{2}}{E_{d} I_{d}},
\end{aligned}
$$

where $L_{d}$ is the total length of dropper, $\rho_{d}$ represents the density of dropper, $v_{x}$ and $v_{y}$, respectively, represent the speeds of motion in the $x$ and $y$ directions, $g$ is the gravity acceleration, $f_{x}, f_{y}$ are the components of the force along the $x$ and $y$ directions, respectively, $E_{d}$ is the elasticity modulus of dropper, $A_{d}$ is the cross-sectional area of dropper, $m$ is the bending moment, and $E_{d} I_{d}$ is the bending rigidity of dropper.

According to the relationships among the definition of strain, arc length, and the coordinates $x, y$, the axial strain of the element caused by the axial tension can be obtained as follows:

$$
e=\frac{F_{N}}{E_{d} A_{d}}=\frac{\mathrm{d} s^{\prime}-\mathrm{d} s}{\mathrm{~d} s} .
$$

The axial tension of the element can be expressed as the following equation:

$$
F_{N}=E_{d} A_{d}\left(\bar{f}_{x} \cos \theta+\bar{f}_{y} \sin \theta\right) .
$$

Further, it can be written as follows:

$$
\frac{\mathrm{d} s^{\prime}-\mathrm{d} s}{\mathrm{~d} s}=\sqrt{\left(\frac{\partial x(s, t))}{\partial s}\right)^{2}+\left(\frac{\partial y(s, t))}{\partial s}\right)^{2}}-1
$$

where $F_{N}$ is the axial tension, $\theta$ is the angle between the micro-element segment and the $x$ direction, $\mathrm{d} s^{\prime}$ is the length of the micro-element segment after the deformation, and $\mathrm{d} s$ is the original length.

Therefore, we can obtain the following expression:

$$
\sqrt{\left(\frac{\partial x(s, t)}{\partial s}\right)^{2}+\left(\frac{\partial y(s, t)}{\partial s}\right)^{2}}-1-\left(\bar{f}_{x} \cos \theta+\bar{f}_{y} \sin \theta\right)=0 .
$$

According to the differential relationship between bending moment and shear force, the following equations can be obtained:

$$
\begin{aligned}
\frac{\partial \theta}{\partial \bar{s}}-\lambda^{2} \bar{m} & =0 \\
\frac{\partial \bar{m}}{\partial \bar{s}}-\left(\bar{f}_{x} \sin \theta-\bar{f}_{y} \cos \theta\right) & =0 .
\end{aligned}
$$

The equations of motion of the microelement segment are expressed as follows:

$$
\begin{array}{r}
\frac{\partial \bar{f}_{x}}{\partial \bar{s}}-u \frac{\partial^{2} \bar{x}}{\partial \bar{t}^{2}}=0, \\
\frac{\partial \bar{f}_{y}}{\partial \bar{s}}-u\left(\frac{\partial^{2} \bar{y}}{\partial \bar{t}^{2}}+1\right)=0 .
\end{array}
$$


The differential relationship between the angle $\theta$ and the coordinates $x, y$ can be obtained:

$$
\frac{\mathrm{d} \bar{y}}{\mathrm{~d} \bar{x}}-\tan \theta=0
$$

All the above equations can be sorted into the following equations:

$$
\left\{\begin{array}{l}
\sqrt{\left(\frac{\partial \bar{x}}{\partial \bar{s}}\right)^{2}+\left(\frac{\partial \bar{y}}{\partial \bar{s}}\right)^{2}}-1=\overline{f_{x}} \cos \theta+\overline{f_{y}} \sin \theta \\
\frac{\partial \theta}{\partial \bar{s}}=\lambda^{2} \bar{m} \\
\frac{\partial \bar{m}}{\partial \bar{s}}=\overline{f_{x}} \sin \theta-\overline{f_{y}} \cos \theta \\
\frac{\partial \overline{f_{x}}}{\partial \bar{s}}=\mu \frac{\partial^{2} \bar{x}}{\partial \bar{t}^{2}} \\
\frac{\partial \overline{f_{y}}}{\partial \bar{s}}=\mu \frac{\partial^{2} \bar{y}}{\partial \overline{t^{2}}}+\mu \\
\frac{\mathrm{d} \bar{y}}{\mathrm{~d} \bar{x}}=\tan \theta
\end{array}\right.
$$

The dropper stress is the sum of the axial and bending stresses; namely,

$$
\sigma=\frac{F_{N}}{A}+\frac{m}{w_{z}}
$$

where $w_{z}$ is the bending section coefficient of dropper.

\subsection{Initial and Boundary Conditions of Each Dropper.} Due to different locations, the initial and boundary conditions of each dropper are different when dropper stress is calculated. They can be expressed as follows:

$$
\begin{aligned}
& \left\{\begin{array}{l}
\bar{x}(\bar{s}, \bar{t})=\frac{10(j-1)}{L_{d}} \\
\bar{y}(\bar{s}, \bar{t})=\bar{s}
\end{array},\right. \\
& \text { when } \bar{t}=0 \text {, } \\
& \left\{\begin{array}{l}
\bar{x}(\bar{s}, \bar{t})=\frac{10(j-1)}{L_{d}} \\
\bar{y}(\bar{s}, \bar{t})=\frac{A_{j}}{L_{d}} \sin (2 \pi \bar{f} \cdot \bar{t})=\bar{A}_{j} \sin (2 \pi \bar{f} \cdot \bar{t})
\end{array}\right. \\
& \left\{\begin{array}{l}
\bar{x}(\bar{s}, \bar{t})=\frac{10(j-1)}{L_{d}}, \\
\bar{y}(\bar{s}, \bar{t}) \leq 1
\end{array} \quad \text { when } \bar{s}=1,\right.
\end{aligned}
$$

where $A_{j}(j=1,2,3,4,5)$ is the vertical displacement amplitude of the $j$ th dropper, $\bar{A}_{j}=\left(A_{j} / L_{d}\right)$ is the dimensionless amplitude, and $\bar{f}=f \sqrt{\left(L_{d} / g\right)}$ is the dimensionless frequency.

3.3. Calculation Parameters of Dropper and Contact Wire. Dropper and contact wire are made of copper stranded wires, and their elastic moduli can be measured by an electronic extensometer (Figure 3).

The bending rigidity is based on Costello's model. In 1997, Costello deduced the calculation formula of the bending rigidity of stranded wire [21]. In the calculation of dropper stress, the specific values of required parameters are shown in Table 1.

3.4. Numerical Method. It is very difficult to find the analytical solution of the equation group (29). Therefore, we can use a finite difference method to find the numerical solution by writing a MATLAB program. A dropper is divided into $n$ equal length elements. The dimensionless length of each element is $\Delta \bar{s}=(1 / n)$, and the dimensionless coordinates of the ith element are $\bar{x}_{i}, \bar{y}_{i}(i=0,1,2, \cdots, n) . \Delta \bar{t}$ is the dimensionless time step and the discretization time is $\bar{t}_{j}=j \Delta \bar{t}(j=0,1,2, \cdots, n)$.

The partial differential equations about the arc length coordinate and time can be expressed approximately as follows:

$$
\begin{aligned}
\frac{\partial \bar{f}}{\partial \bar{s}} & =\frac{\bar{f}_{i+1}-\bar{f}_{i}}{\Delta \bar{s}}, \\
\frac{\mathrm{d} \bar{y}}{\mathrm{~d} \bar{x}} & =\frac{\bar{y}_{i+1}-\bar{y}_{i}}{\bar{x}_{i+1}-\bar{x}_{i}}, \\
\frac{\partial^{2} \bar{g}}{\partial \bar{t}^{2}} & =\frac{\bar{g}_{j+2}-2 \bar{g}_{j+1}+\bar{g}_{j}}{\Delta \bar{t}^{2}},
\end{aligned}
$$

where $\bar{f}, \bar{g}$ are the functions of the dimensionless arc length coordinate $\bar{s}$ and the dimensionless time $\bar{t}$.

Undoubtedly, the number of discrete elements and the time step affect the accuracy of numerical solution. The larger the number of discrete elements and the smaller the time step, the higher the accuracy of numerical solution, but the total calculation time also increases. The number of elements $n=32$ and the dimensionless time step $\Delta \bar{t}=7.5 \times$ $10^{-10}$ are selected in the numerical calculation, which not only meets the required accuracy but also economizes the calculation time.

\section{Results}

To investigate the effects of load location on dropper stress, we select two special sites. One is the edge and the other is the middle of contact wire. When a load acts on the edge of contact wire, the value of $a$ in Figure 1 is 0 . By substituting $x=0,10,20,30,40$ into the response equation of the beam, the vertical displacement of each dropper can be obtained: 


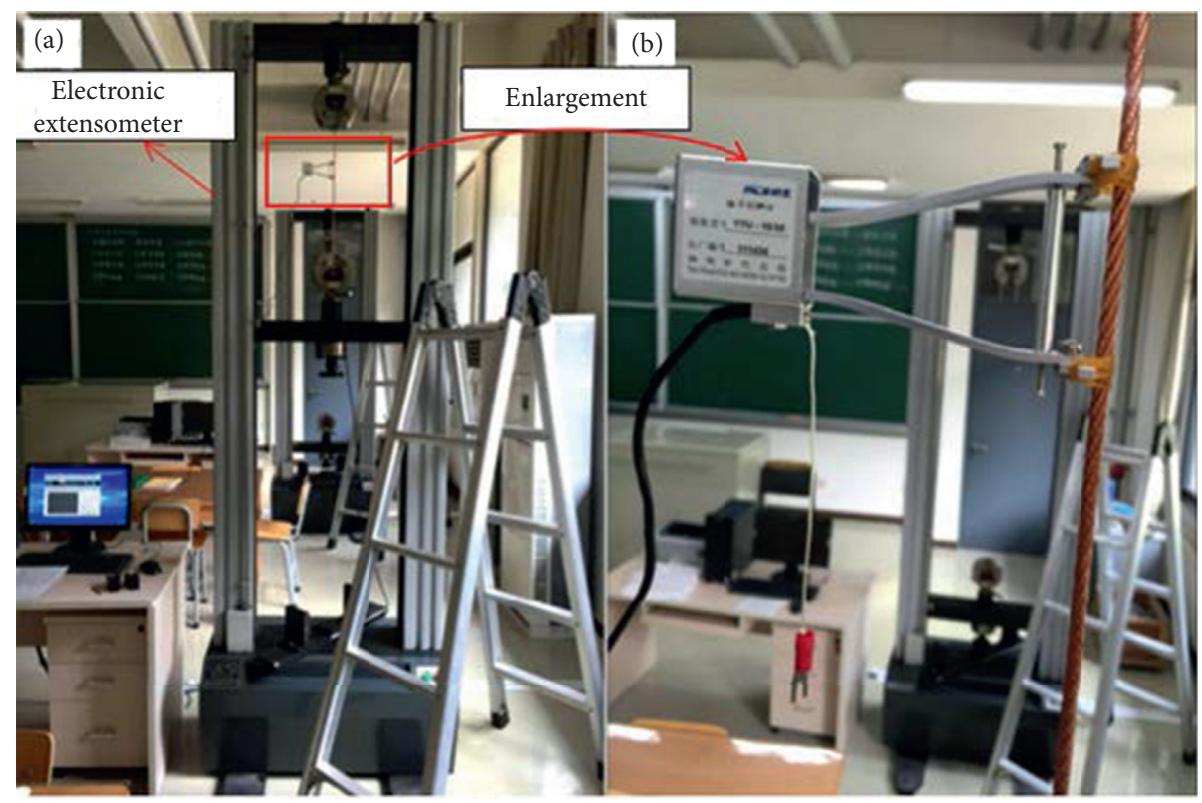

FIgUre 3: The parameter test of materials.

TABLE 1: Dropper and contact wire parameters.

\begin{tabular}{lccccc}
\hline & Density $\left(\mathrm{kg} \cdot \mathrm{m}^{-3}\right)$ & Cross-sectional area $\left(\mathrm{m}^{2}\right)$ & Elastic modulus $(\mathrm{Pa})$ & Bending rigidity $\left(\mathrm{Pa} \cdot \mathrm{m}^{4}\right)$ & $\begin{array}{c}\text { Bending section } \\
\text { coefficient }\left(\mathrm{m}^{3}\right)\end{array}$ \\
\hline Dropper & $8.9 \times 10^{3}$ & $1.29 \times 10^{-5}$ & $83.29 \times 10^{9}$ & 2.53 & $6.7663 \times 10^{-9}$ \\
Contact wire & $8.9 \times 10^{3}$ & $1.58 \times 10^{-4}$ & $118.49 \times 10^{9}$ & 233.92 & - \\
\hline
\end{tabular}

$$
\left\{\begin{array}{l}
y(0, t)=0.053 \sin 2 \pi t, \\
y(10, t)=0.012 \sin 2 \pi t, \\
y(20, t)=0.009 \sin 2 \pi t, \\
y(30, t)=0.004 \sin 2 \pi t, \\
y(40, t)=0.002 \sin 2 \pi t .
\end{array}\right.
$$

Similarly, when a load acts in the middle of contact wire, the value of $a$ is 20. Thus, the vertical displacement of each dropper can be obtained:

$$
\left\{\begin{array}{l}
y(0, t)=0.006 \sin 2 \pi t, \\
y(10, t)=0.007 \sin 2 \pi t, \\
y(20, t)=0.014 \sin 2 \pi t, \\
y(30, t)=0.007 \sin 2 \pi t, \\
y(40, t)=0.006 \sin 2 \pi t .
\end{array}\right.
$$

4.1. Dropper Stress under the Load Acting on the Edge of Contact Wire. When the load acts on the edge of contact wire, it causes the vibration of contact wire, and dropper also vibrates under the influence of contact wire, which causes the fluctuation of dropper stress. The maximum tensile and compressive stresses are induced in a very short time. There are three stages during the period of the stress changes, including instant rebound, damped sinusoidal vibration, and bending compression (Figure 4). The first stage is caused when dropper I deforms instantaneously from compression to tension, and the stress changes suddenly to the maximum from the value of nearly zero; then, compression emerges by the inertia. At the second stage, the dropper behavior is similar to a spring oscillator. The dropper is in a tiny bending state at the third stage. The reason is that the bending stiffness of dropper is very small; the stress is almost zero. When dropper is transitioning from one stage to another, it is subjected to an impact force.

The stress changes of dropper II also experience three stages (Figure 5). It is the same as the vibration form of dropper I, but the maximum tensile stress is only half of that of dropper I, and the maximum compressive stress is lower, and the time to reach the maximum tensile stress is longer. The reason is that the vibration of dropper I is caused and it spreads to the surrounding in the form of waves, and then the vibration of the adjacent dropper is produced when the load is applied to the edge of contact wire. Since the energy loss occurs in the process of wave propagation, the vibration amplitude of the surrounding dropper is reduced. The maximum tensile stress of dropper II needs a longer time to reach because the wave propagation spends time. 


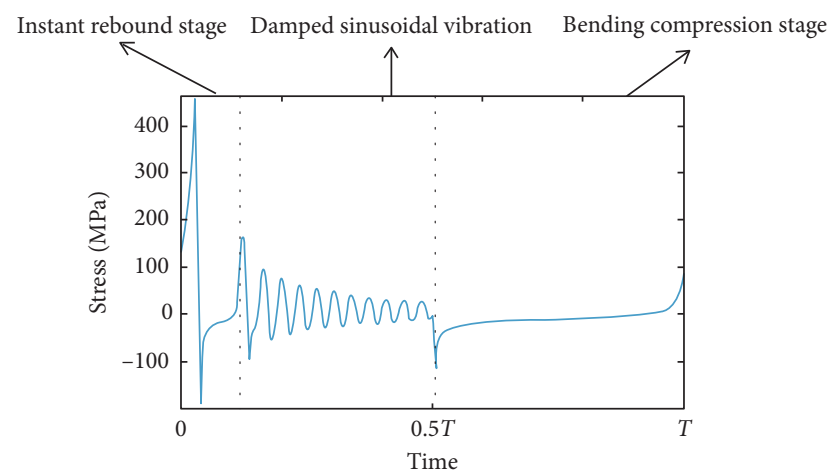

FIgURE 4: The stress changes of dropper I with time.

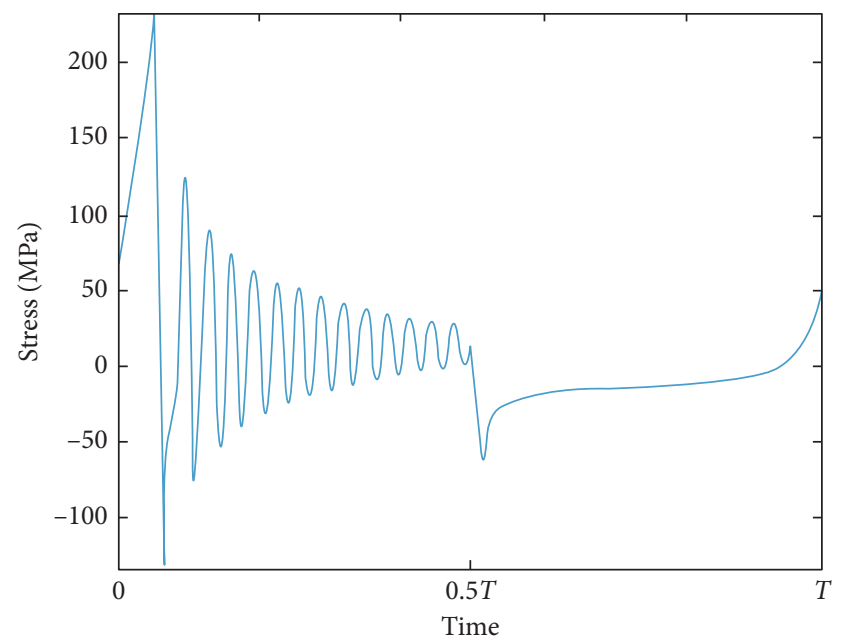

Figure 5: The stress changes of dropper II with time.

Compared with droppers I and II, the time is longer when the vibration amplitude of dropper III reaches the maximum. This is because the distance between droppers III and I is far, and the spent time in the process of wave propagation is longer. The vibration amplitude of dropper III is lower than that of dropper II with the decrease of energy (Figure 6).

The stress of dropper IV fluctuates under the influence of the load. Compared with droppers I, II, and III, the vibration and stress change amplitude are greatly weakened, and the time to reach the maximum tensile and compressive stresses is longer, which is caused by the location of the dropper (Figure 7).

Compared with other droppers in a span, the vibration amplitude of dropper $\mathrm{V}$ is the lowest, and its maximum tensile and compressive stress values are also the lowest. The reason for this result is that dropper $\mathrm{I}$ is the acting location of the load and dropper $\mathrm{V}$ is the farthest from dropper I. That is, the farthest distance from the wave source results in the lowest vibration amplitude and the weakest stress fluctuation of dropper $\mathrm{V}$. The time to reach the maximum tensile and compressive stresses is the longest, and the number of attenuations is the least (Figure 8).

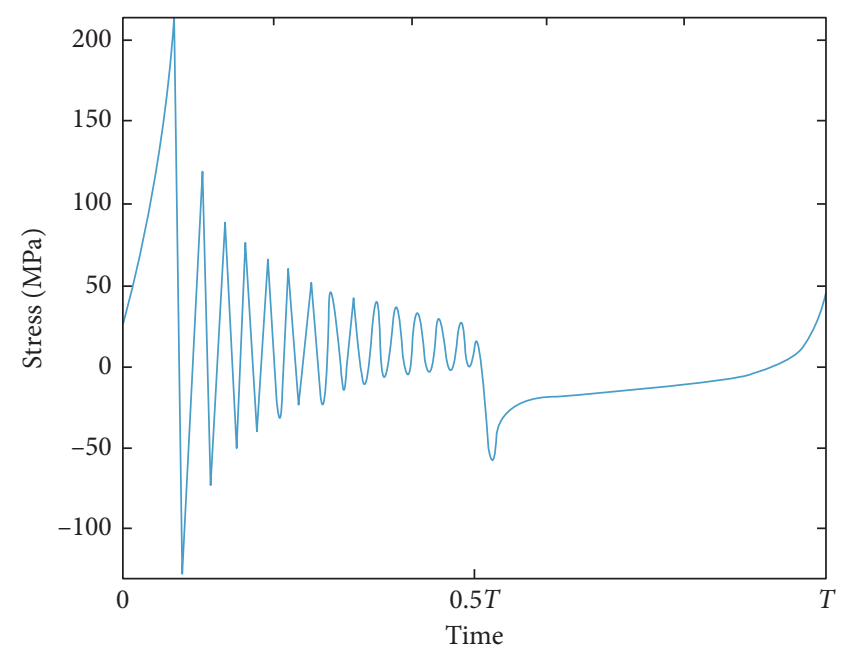

FIgURE 6: The stress changes of dropper III with time.

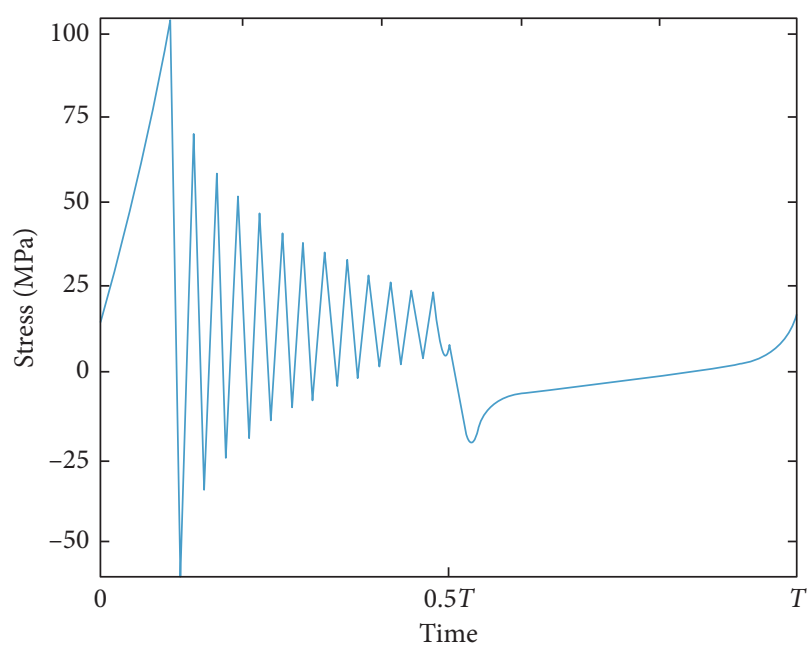

Figure 7: The stress changes of dropper IV with time.

4.2. Dropper Stress under the Load Acting in the Middle of Contact Wire. This model in this work has the characteristics of symmetry about dropper III; only the stresses of droppers I, II, and III need to be calculated when the load acts in the middle of contact wire. It can be seen from Figure 9 that the stress changes of dropper I go through three stages in a cycle. Due to the symmetry of this model, the stress characteristics of droppers I and V are consistent.

Compared with dropper I, the maximum tensile and compressive stresses of dropper II are increased and the time to reach the maximum values is decreased. This is because the location of each dropper in a catenary system is different, which results in different amplitude of each dropper in a span. Dropper II is closer to dropper III; thus, it vibrates earlier than dropper I. Therefore, in a cycle, dropper II spends a shorter time in the rebound stage, and the attenuation is relatively longer (Figure 10).

Figure 11 shows that the stress changes of dropper III are the most severe within the span, and the number of 


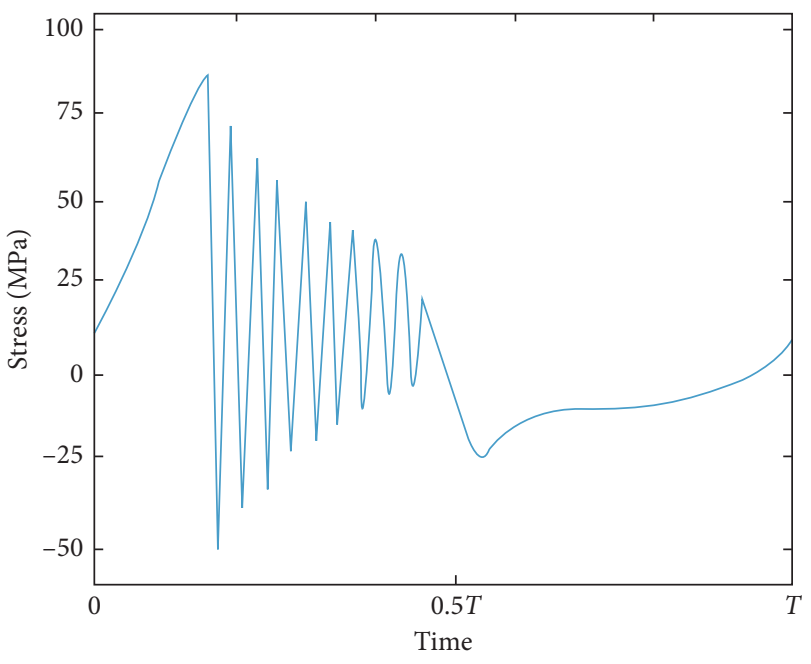

FIgURE 8: The stress changes of dropper $\mathrm{V}$ with time.

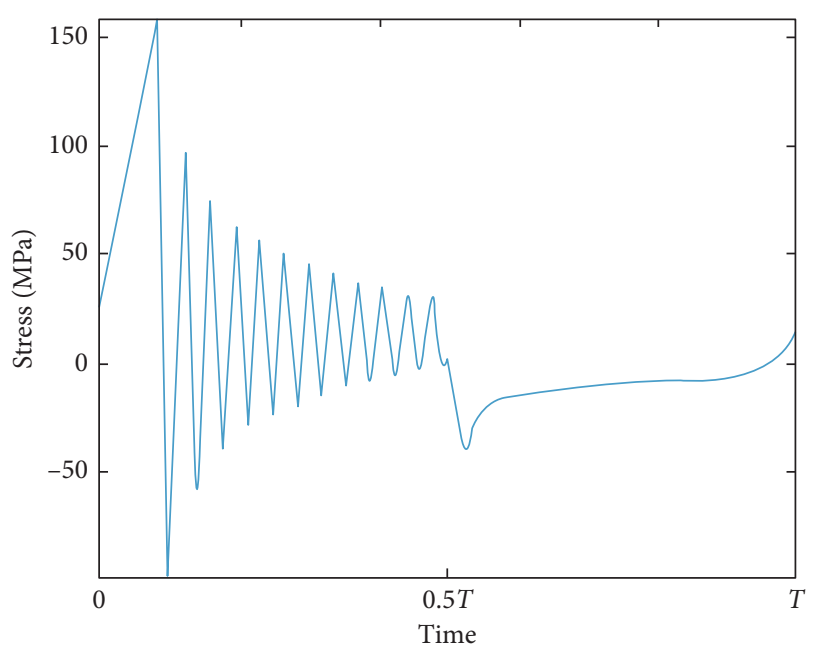

Figure 9: The stress changes of dropper I with time.

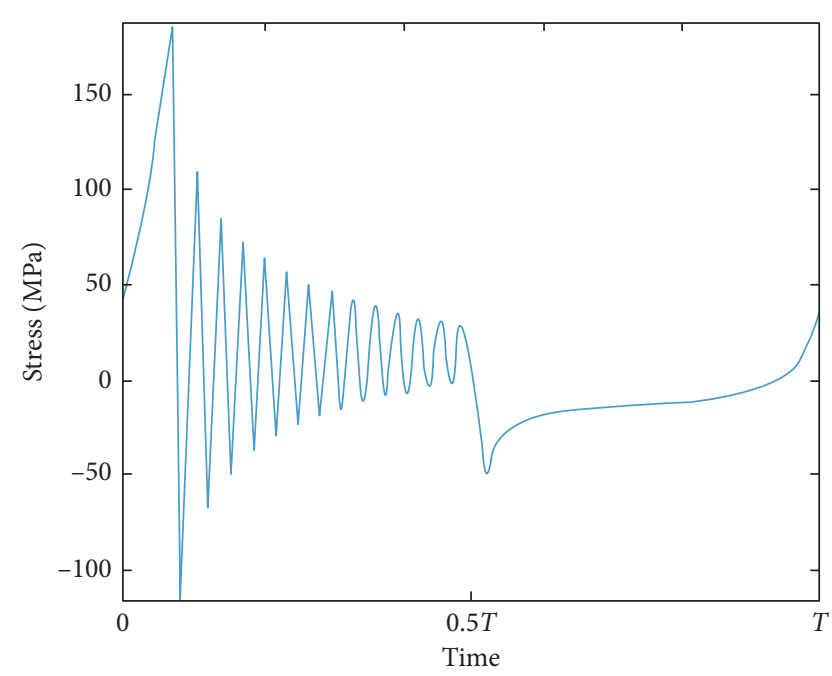

Figure 10: The stress changes of dropper II with time.

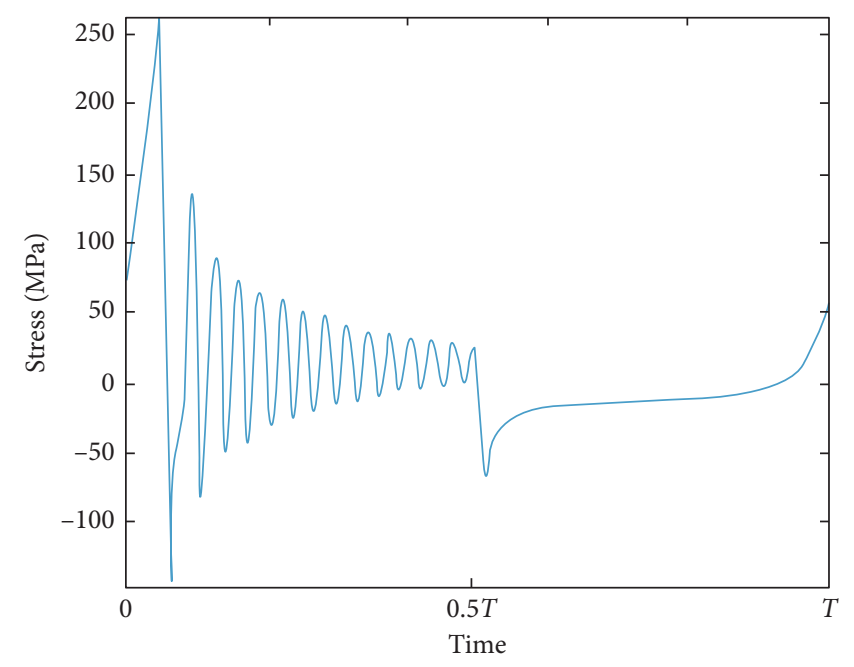

FIgURE 11: The stress changes of dropper III with time.

attenuations is the most. The time to reach the maximum tensile and compressive stresses is the least.

\section{Discussion}

During a period, the dropper stress changes with time experience three stages, namely, instant rebound, damped sinusoidal, and bending compression stages. This is because dropper vibrates under a load, which makes the original dropper in the straightened state suffer a large impact force. When dropper vibrates and lifts, it is in a relaxed state and then returns to the original state under the gravity. In this paper, the vibration of dropper under the actual working condition is equivalent to the forced vibration caused by the simple harmonic vibration of the connection point (driving point) between dropper and the contact wire. Firstly, due to the influence of its own gravity, dropper is in a straight state and keeps a low tensile stress. When a load acts on contact wire, the driving point vibrates in the form of the simple harmonic vibration. The driving point moves downward, and dropper is always in a straight state. Then, when the driving point moves upward rapidly, dropper loosens and bends. In the process of the forced vibration caused by the driving point, the tensile stress increases when dropper is straightened. However, the tensile stress decreases and the compressive stress increases during the bending. In the process of the vibration, the driving point moves back and forth, and the dropper stress changes maintain the same in a cycle.

No matter where a load acts on contact wire, the tensile and compressive stresses of dropper closest to the load change most obviously. The farther the dropper is away from the load, the smaller the stress change range. The reason is that the driving point causes the vibration of dropper and the vibration spreads to other locations. However, due to the loss of energy with the increase of the distance from the load location, the vibration amplitude gradually decreases. It can be seen that the stress changes of dropper are related to the 


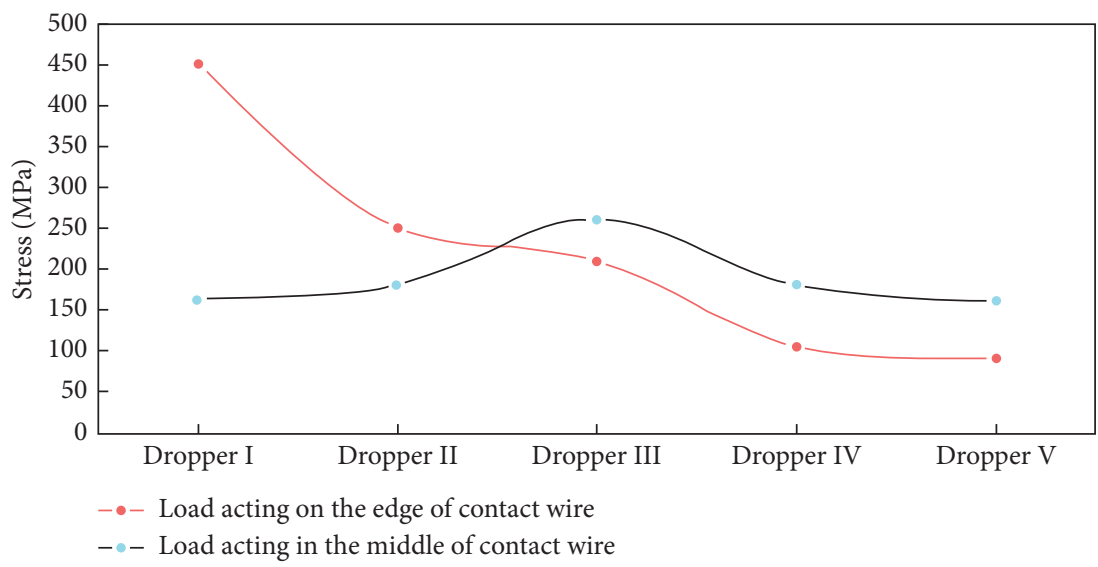

FIgURE 12: Comparison of the maximum tensile stress of each dropper.

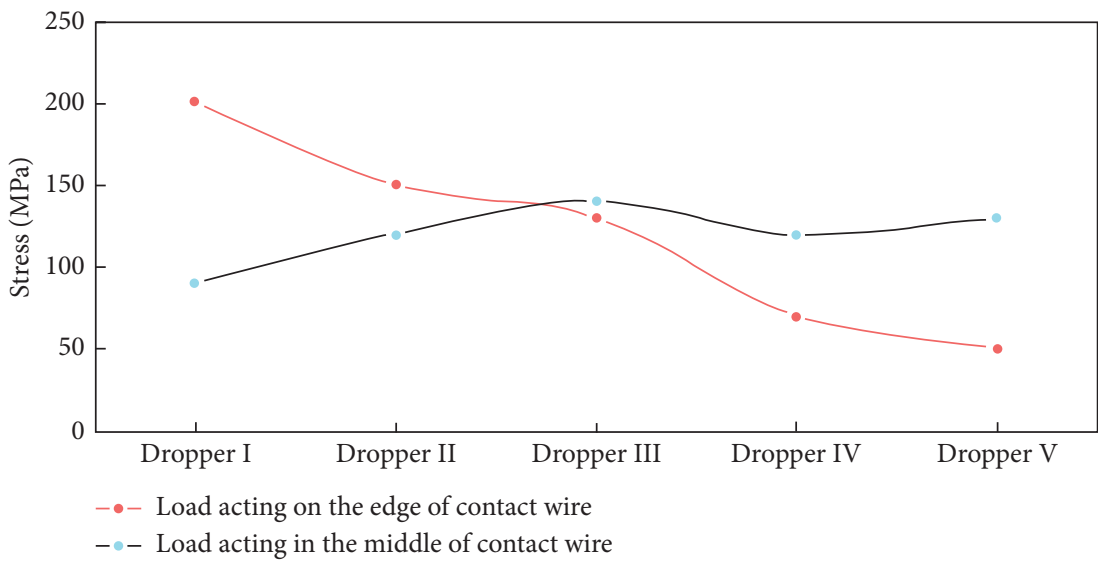

Figure 13: Comparison of the maximum compressive stress of each dropper.

load location. The closer the distance is, the stronger the vibration is, and the larger the stress change range is.

The relationships between the maximum tensile and compressive stresses of each dropper and its location are shown in Figures 12 and 13. When the load acts on the edge of contact wire, the maximum tensile and compressive stresses and the change amplitude are larger than those under the load acting in the middle of contact wire. It can be seen that the load acting on different locations has a significant impact on dropper stress, and the stress changes of dropper at the edge are more obvious. Chen analyzed the dynamic force of dropper and obtained the compression amplitude of droppers I and V by measuring the lifting amount of dropper through an experiment and treating it as an initial load of finite element analysis to simulate the situation of pantograph acting on catenary. Chen's results are consistent with ours [22]. The results indicate that the dropper at the edge is more prone to fatigue damage. Therefore, it is necessary to add supports at both ends.

\section{Conclusion}

This work discusses the effects of load location on dropper stress in a catenary structure. The stress equation for dropper is presented. Both the numerical and analytical models have been studied. The conclusions can be drawn as follows:

(1) The stress changes of dropper under a load can be roughly divided into three stages: instant rebound, damped sinusoidal vibration, and bending compression. The shorter the distance away from the load is, the larger the vertical displacement of dropper is, which results in the corresponding increases of its stress amplitude and the maximum tensile stress.

(2) The load location has a significant impact on the stress changes of dropper. Compared to the condition of the load in the middle, the load acting on the edge of contact wire could induce the larger tensile stress when both ends of contact wire are considered as free boundaries.

\section{Data Availability}

The data used to support the findings of this study are included within the article.

\section{Conflicts of Interest}

The authors declare that they have no conflicts of interest. 


\section{Acknowledgments}

The authors acknowledge the Key Project of Technological Research and Development Plan of China Railway Corporation (2017J010-A) for financially supporting this work.

\section{References}

[1] Y. H. Cho, "Numerical simulation of the dynamic responses of railway overhead contact lines to a moving pantograph, considering a nonlinear dropper," Journal of Sound and Vibration, vol. 315, no. 3, pp. 433-454, 2008.

[2] O. Lopez-Garcia, A. Carnicero, and J. L. Marono, "Influence of stiffness and contact modelling on catenary-pantograph system dynamics," Journal of Sound and Vibration, vol. 299, no. 4-5, pp. 806-821, 2007.

[3] M. Aboshi and K. Manabe, "Analyses of contact force fluctuation between catenary and pantograph," Quarterly Report of RTRI, vol. 41, no. 4, pp. 182-187, 2000.

[4] D. S. Zhang and M. Ostoja-Starzewski, "Finite element solutions to the bending stiffness of a single-layered helically wound cable with intemal friction," Journal of Applied Mechanics, vol. 83, no. 3, Article ID 031003, 2016.

[5] J. F. Sun, G. L. Wang, and H. O. Zhang, "FE analysis of frictional contact effect for laying wire rope," Journal of Materials Processing Technology, vol. 202, no. 1-3, pp. 170178,2008

[6] W.-G. Jiang, "A concise finite element model for pure bending analysis of simple wire strand," International Journal of Mechanical Sciences, vol. 54, no. 1, pp. 69-73, 2012.

[7] M. L. Yu, W. Z. Liu, J. Zhang, and C. Y. Yan, "Influence of dropper spacing on quality of pantograph-catenary current collection," Applied Mechanics and Materials, vol. 654, pp. 78-81, 2014.

[8] L. M. Chen, P. H. Peng, and F. He, "Fatigue life analysis of dropper used in pantograph-catenary system of high-speed railway," Advances in Mechanical Engineering, vol. 10, no. 5, 2018.

[9] W. Zhang, G. Mei, and J. Zeng, "A study of pantograph/ catenary system dynamics with influence of presag and irregularity of contact wire," Vehicle System Dynamics, vol. 37, no. sup1, pp. 593-604, 2016.

[10] J. Ambrósio, J. Pombo, F. Rauter, and M. Pereira, "A memory based communication in the co-simulation of multibody and finite element codes for pantograph-catenary interaction simulation," Multibody Dynamics, vol. 12, pp. 231-252, 2009.

[11] A. Collina and S. Bruni, "Numerical simulation of pantograph-overhead equipment interaction," Vehicle System Dynamics, vol. 38, no. 4, pp. 261-291, 2010.

[12] S. Bruni, J. Ambrosio, A. Carnicero et al., "The results of the pantograph-catenary interaction benchmark," Vehicle System Dynamics, vol. 53, no. 3, pp. 412-435, 2015.

[13] M. Tur, E. García, L. Baeza, and F. J. Fuenmayor, “A 3D absolute nodal coordinate finite element model to compute the initial configuration of a railway catenary," Engineering Structures, vol. 71, pp. 234-243, 2014.

[14] O. Lopez-Garcia, A. Carnicero, V. Torres, and J. R. JimenezOctavio, "The influence of cable slackening on the stiffness computation of railway overheads," International Journal of Mechanical Sciences, vol. 50, no. 7, pp. 1213-1223, 2008.

[15] A. Andreu, L. Gil, and P. Roca, "A new deformable catenary element for the analysis of cable net structures," Computers \& Structures, vol. 84, no. 29-30, pp. 1882-1890, 2006.
[16] M. Such, J. R. Jimenez-Octavio, A. Carnicero, and O. LopezGarcia, "An approach based on the catenary equation to deal with static analysis of three dimensional cable structures," Engineering Structures, vol. 31, no. 9, pp. 2162-2170, 2009.

[17] Y. Chen, J. Feng, R. J. Ma, and Y. T. Zhang, "Efficient symmetry method for calculating integral prestress modes of statically indeterminate cable-strut structures," Journal of Structural Engineering, vol. 141, no. 10, Article ID 04014240, 2015.

[18] Y. Chen, J. Y. Yan, P. Sareh, and J. Feng, "Feasible prestress modes for cable-strut structures with multiple self-stress states using particle swarm optimization," Journal of Computing in Civil Engineering, vol. 34, no. 3, Article ID 04020003, 2020.

[19] A. Zingoni, "Group-theoretic exploitations of symmetry in computational solid and structural mechanics," International Journal for Numerical Methods in Engineering, vol. 79, no. 3, pp. 253-289, 2009.

[20] Y. Chen, P. Sareh, J. Feng, and Q. Sun, "A computational method for automated detection of engineering structures with cyclic symmetries," Computers \& Structures, vol. 191, pp. 153-164, 2017.

[21] G. A. Costello, Theory of Wire Rope, pp. 1-50, Springer, New York, NY, USA, 1997.

[22] L. M. Chen, "Study on dynamic force of integral dropper of catenary under action of high-speed pantograph," China Railway Science, vol. 39, no. 3, pp. 86-92, 2018. 